\title{
Dual Solutions of Casson nanofluid flow due to an Exponentially porous Stretching Surface with a Second-order Slip: Stability Analysis
}

\author{
Debasish Dey ${ }^{1}$ and RUPJYOTI BORAH ${ }^{1}$ \\ ${ }^{1}$ Dibrugarh University
}

June 20, 2021

\begin{abstract}
An analysis has been done to scrutinize the existence of dual solutions of the Casson nanofluid caused due to an exponential form of stretching surface which is situated in porous medium and second order velocity slip. An uniform magnetic field is considered in the transverse dirction of the flow. A suitable similarity transformation is employed to amend the model equations into solvable form and hence solved by adopting the MATLAB built-in bvp4c solver scheme. Results are discussed through both graphical mode and tabulations with respect to some novel flow parameters. The stability analysis has been studied and found two solutions, one is stable in nature and physically tractable. From the study, we have seen that the flow parameters associated with nanofluid enhance the heat transfer of the fluid. The Casson fluid parameter has also a power to increase temperature of the system.
\end{abstract}

\section{Hosted file}

Dey and Borah Manuscript.doc available at https://authorea.com/users/420655/articles/526958dual-solutions-of-casson-nanofluid-flow-due-to-an-exponentially-porous-stretchingsurface-with-a-second-order-slip-stability-analysis 